\title{
Gene expression levels of cytokines in peripheral blood mononuclear cells from patients with pulmonary embolism
}

\author{
WEI LV*, QIANGLIN DUAN*, LEMIN WANG, ZHU GONG, FAN YANG and YANLI SONG \\ Department of Cardiology, Tongji Hospital, Tongji University School of Medicine, Shanghai 200065, P.R. China
}

Received November 15, 2012; Accepted February 18, 2013

DOI: $10.3892 / \mathrm{mmr} .2013 .1344$

\begin{abstract}
The aim of this study was to investigate the differential gene expression of cytokines in peripheral blood mononuclear cells (PBMCs) from patients with pulmonary embolism (PE) and controls. Twenty patients with PE and twenty control patients matched for gender and age with the PE group were recruited into the study. Human cDNA microarray analysis was used to detect differences in the expression of cytokineassociated genes between the two groups. In PE patients, the expression levels of the genes encoding IFN $\alpha 5$, IFN $\alpha 6$, IFN $\alpha 8$, IFN $\alpha 14$, IFN $\kappa$, IFN $\omega 1$, IFN $\varepsilon 1$ and IFN $\gamma$ were significantly lower compared with controls $(\mathrm{P}<0.05)$. The expression levels of the genes encoding IL1 $\alpha$, IL2, IL3, IL9, IL13, IL17 $\beta$, IL19, IL22, IL23 $\alpha$, IL24, IL25 and IL31 were significantly lower $(\mathrm{P}<0.05)$, while IL10 and IL28A mRNA expression levels were higher in $\mathrm{PE}$ patients compared with controls $(\mathrm{P}<0.05)$. In $\mathrm{PE}$ patients, $\mathrm{Cxcl1}, \mathrm{Cxcl} 2, \mathrm{Cxcl} 6, \mathrm{Cxcl13}$ and $\mathrm{Cxcl14}$ mRNAs were significantly upregulated $(\mathrm{P}<0.05)$, however, Cxcl10 mRNA was significantly downregulated $(\mathrm{P}<0.01)$. In $\mathrm{PE}$ patients, the mRNA expression levels of TNF superfamily members 1,9 and 13, and TNF receptor superfamily members $1 \mathrm{~A}, 1 \mathrm{~B}, 9,10 \mathrm{~B}, 10 \mathrm{C}$, $10 \mathrm{D}$ and $19 \mathrm{~L}$, were significantly upregulated $(\mathrm{P}<0.05)$, whereas TNF receptor superfamily members $11 \mathrm{~B}, 19$ and 25 were significantly downregulated compared with controls $(\mathrm{P}<0.05)$. The mRNA expression levels of granulocyte-macrophage colony-stimulating factor, granulocyte colony-stimulating factor, erythropoietin, thrombopoietin and mast cell growth factor were significantly lower in PE patients compared with controls $(\mathrm{P}<0.05)$. In PE patients, the mRNA expression levels of a variety of cytokines were imbalanced and cellular immune function was downregulated compared with controls. Thus, PE patients may be more susceptible to infections caused by viruses, intracellular bacteria and parasites.
\end{abstract}

Correspondence to: Professor Lemin Wang, Department of Cardiology, Tongji Hospital, Tongji University School of Medicine, 389 Xincun Road, Shanghai 200065, P.R. China

E-mail: wanglemin@tongji.edu.cn

*Contributed equally

Key words: pulmonary embolism, cytokine, mRNA, peripheral blood mononuclear cells

\section{Introduction}

Deep vein thrombosis (DVT) and pulmonary embolism (PE) are collectively known as venous thromboembolism (VTE). PE includes acute pulmonary embolism (APE) and chronic thromboembolic pulmonary hypertension (CTEPH). PE is a major health problem, with a high incidence, mortality, misdiagnosis and low detection rate (1). There is much debate with regard to the diagnosis, treatment and prevention of $\mathrm{PE}$, as the etiology and pathogenesis of this disease have not been well characterized. It has been reported that patients with APE and CTEPH possess lowered immune function $(2,3)$. In this study, we used human cDNA microarray analysis to determine the mRNA expression levels of cytokines in PBMCs isolated from PE patients and controls. We designed this in vitro study to observe changes in the immune function of PE patients.

\section{Patients and methods}

Patients. Twenty patients with PE were recruited from the Tongji Hospital (Tongji University School of Medicine, Shanghai, China) between 2007 and 2008. A diagnosis of PE required any two of the following three criteria to be met: i) selective pulmonary angiography demonstrating pulmonary artery obstruction or filling defect; ii) lung ventilation/perfusion scan showing single or multiple blood perfusion defects, normal or; iii) clinical diagnosis: there are risk factors for PE and other cardiovascular diseases that are able to be excluded by clinical performance, electrocardiogram and chest film. Arterial blood gas analysis suggested that hypoxemia and hypocapnia, D-dimer detection, echocardiography and chest computed tomography support the diagnosis of PE. We selected 20 patients admitted to the Department of Cardiology, Tongji Hospital, at the same time as the controls used in the study. The patients were divided into two groups: i) the PE patient group: 20 patients (11 males and 9 females), with a mean age of $70 \pm 14$ years (range, 44-89 years), including 3 cases of CTEPH; ii) the control group: 20 patients (11 males and 9 females) without PE, DVT, arterial thrombosis and congenital coagulation abnormality, with a mean age of $72 \pm 14$ years (range, $44-91$ years), who were matched for gender and age with the PE group. There was no significant statistical difference between the age of the two groups $(\mathrm{P}>0.05)$. The clinical trial was approved by the Ethics Committee of the Tongji University and written informed consent was obtained. 
Gene expression profiling. Agilent G4112A Whole Human Genome Oligo Microarrays were purchased from Agilent Technologies (Palo Alto, CA, USA). The microarray was composed of 44,290 spots, including 41,675 genes or transcripts, 314 negative control spots, 1,924 positive control spots and 359 blank spots. The functions of $>70 \%$ of the genes in the microarray were known. All patients were subjected to microarray analysis.

Total RNA isolation. A 5-ml sample of peripheral blood anticoagulated with EDTA was drawn from patients suspected with PE immediately following hospital admittance and from those patients without PE. Mononuclear cells were obtained through density gradient centrifugation $(3,000 \mathrm{x}$ g) with Ficoll solution and the remaining red blood cells were destroyed with erythrocyte lysis buffer (Qiagen, Hilden, Germany). Total mononuclear cell RNA was extracted with TRIzol (Invitrogen Life Technologies, Carlsbad, USA) and purified with RNeasy column (Qiagen) according to the manufacturer's instructions. Isolated total RNA was evident and quantified by means of Nanodrop ND-1000 spectrophotometer (Nanodrop Technology, Cambrige, UK).

Detection of gene expression. Approximately $1 \mu \mathrm{g}$ of total RNA was reverse transcribed into double-strand cDNA. Following purification, in vitro amplification was performed using the Low RNA Input Linear Amplification kit (Agilent Technologies) and modified UTP [aaUTP, 5-(3-aminoallyl)UTP] was used to replace UTP. The integrated aaUTP is capable of interacting with the Cy3 NHS ester-forming fluorescent products, which are then used for hybridization. The integration rate of fluorescence was determined using a Nanodrop ND-1000 spectrophotometer. Following this, the hybridization mixture was prepared with the oligonucleotide microarray in situ Hybridization Plus kit (Agilent Technologies). Approximately $750 \mathrm{ng}$ of fluorescent products were fragmented at $60^{\circ} \mathrm{C}$ and hybridization was conducted in Human Whole-Genome 60-mer oligo-chips (G4112F, Agilent Technologies) at $60^{\circ} \mathrm{C}$ for $17 \mathrm{~h}$ at $10 \mathrm{rpm}$. Following hybridization, the chips were washed with Gene Expression Wash Buffer (Agilent Technologies) according to the manufacturer's instructions. Original signals were obtained using Scanner and Feature Extraction software (Agilent Technologies). The standardization of original signals was performed with RMA standardized methods and standardized signal values were used for the screening of differentially expressed genes.

$R T-P C R$. Three differentially expressed genes in the microarray analysis were selected and their expression was confirmed by RT-PCR. Among the genes with differential expression, three genes were randomly selected and subjected to RT-PCR along with the housekeeping gene (GAPDH). The relative expression was indicated as the expression of the target genes normalized by that of GAPDH $\left(2^{-\Delta \Delta C t}\right)$. Melting curve and $2^{-\Delta \Delta C t}$ methods were used to compare differences in gene expression between the control and PE groups. Results from RT-PCR were consistent with those obtained from the microarray analysis.

Statistical analysis. The independent samples t-test was used to compare mRNA levels in samples from PE patients and controls.
Statistical tests were performed using SPSS 17.0, and $\mathrm{P}<0.05$ was considered to indicate a statistically significant result. Prior to the t-test, a test for equality of variances was performed; if variances were not equal, the t-test result was corrected.

\section{Results}

IFN mRNA expression levels. PBMCs obtained from PE patients demonstrated low IFN mRNA expression levels. Expression of the 12 genes encoding type I IFNs, as well as the gene encoding IFN $\gamma$, were examined (Fig. 1). In PBMCs from $\mathrm{PE}$ patients, the expression levels of genes encoding IFN $\alpha 5$, IFN $\alpha 6$, IFN $\alpha 8$, IFN $\alpha 14$, IFN $\kappa$, IFN $\omega 1$ and IFN 1 were significantly lower than those detected in PBMCs from controls $(\mathrm{P}<0.05)$. IFN $\gamma$ mRNA expression was significantly downregulated in PBMCs from PE patients compared with controls $(\mathrm{P}<0.01)$.

IL mRNA expression levels. The expression levels of 37 genes encoding ILs were examined (Fig. 2). The expression levels of genes encoding IL1 $\alpha$, IL2, IL3, IL9, IL13, IL17 $\beta$, IL19, IL22, IL23 $\alpha$, IL24, IL25 and IL31 were significantly lower in PBMCs from PE patients compared with the controls $(\mathrm{P}<0.05)$. IL10 and IL28A gene expression levels were greater in PBMCs from $\mathrm{PE}$ patients than controls $(\mathrm{P}<0.05)$.

Th1/Th2 mRNA expression levels. Th1 cytokine mRNA expression levels including IFN $\gamma$ and IL2 mRNAs were detected (Fig. 3), and in PE patients, both were significantly downregulated compared with the control group $(\mathrm{P}<0.01)$. Th2 cytokine mRNA expression levels, including IL4, IL6 and IL10 mRNAs were examined (Fig. 4). IL10 mRNA expression levels were significantly higher in PBMCs from PE patients than controls $(\mathrm{P}<0.01)$. No significant difference in IL4 and IL6 mRNA expression levels was observed between the two groups.

Chemokine mRNA expression levels. Twelve genes encoding CXC chemokines were detected (Fig. 5). In PE patients, mRNA expression levels of Cxcl1, $\mathrm{Cxcl} 2, \mathrm{Cxcl} 6, \mathrm{Cxcl13}$ and $\mathrm{Cxcl} 14$ were significantly upregulated $(\mathrm{P}<0.05)$, and Cxcl10 mRNA expression levels were significantly downregulated compared with controls $(\mathrm{P}<0.01)$. Twenty-three genes encoding CC chemokines were examined (Figs. 6 and 7) and the mRNA expression levels of CC chemokines were significantly lower in PE patients than controls $(\mathrm{P}<0.01)$. $\mathrm{C}$ chemokine mRNA expression levels including Xcl1 and Xcl2 mRNAs were detected (Fig. 8), and Xcl1 mRNA expression was significantly lower in PE patients $(\mathrm{P}<0.01)$. No significant difference in $\mathrm{Xcl} 2 \mathrm{mRNA}$ expression levels was observed between the two groups. CX3C chemokine Cx3cl1 mRNA expression was detected, and there was no significant difference between the two groups.

TNF superfamily and TNF receptor superfamily $m R N A$ expression levels. Thirty-eight genes encoding members of the TNF superfamily and TNF receptor superfamily were examined (Fig. 9). In PE patients, the mRNA expression levels of TNF superfamily members 1,9 and 13, and TNF receptor superfamily members $1 \mathrm{~A}, 1 \mathrm{~B}, 9,10 \mathrm{~B}, 10 \mathrm{C}, 10 \mathrm{D}$ and 19L, were significantly upregulated $(\mathrm{P}<0.05)$, whereas TNF 


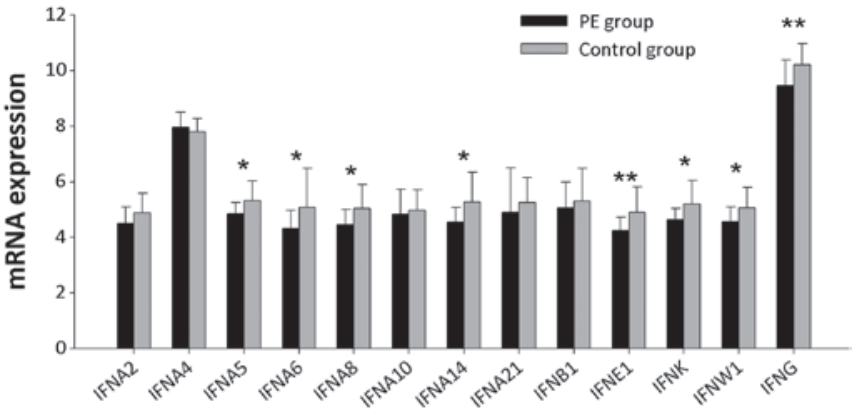

Figure 1. IFN mRNA expression levels in PBMCs from PE patients and controls. IFNA2, IFNA4, IFNA5, IFNA6, IFNA8, IFNA10, IFNA14, IFNA21, IFNB1, IFNE1, IFNK, IFNW1 and INFG represent the gene symbols of IFN $\alpha 2$, IFN $\alpha 4$, IFN $\alpha 5$, IFN $\alpha 6$, IFN $\alpha 8$, IFN $\alpha 10$, IFN $\alpha 14$, IFN $\alpha 21$, IFN $\beta 1$, IFN $\varepsilon 1$, IFN $\chi$, IFN $\omega 1$ and IFN $\gamma$, respectively. ${ }^{*} \mathrm{P}<0.05$ and ${ }^{* * *} \mathrm{P}<0.01$. PBMCs, peripheral blood mononuclear cells; PE, pulmonary embolism.

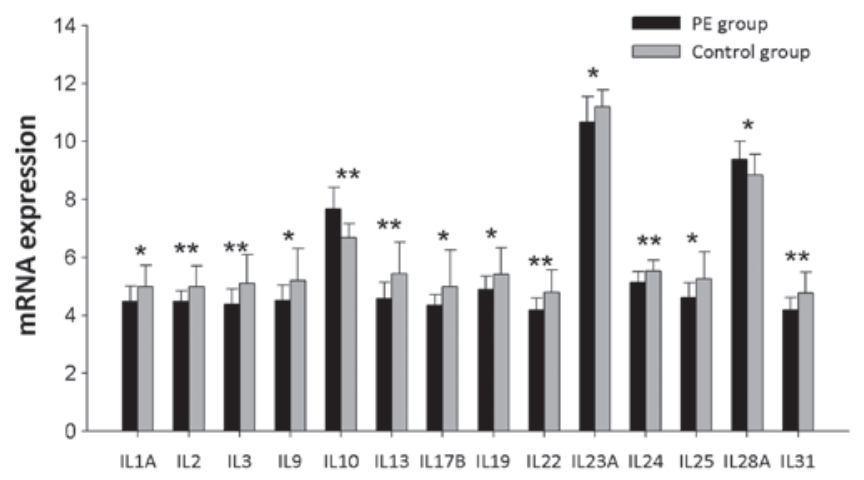

Figure 2. IL mRNA expression levels were significantly different in PBMCs from PE patients and controls. IL1A, IL2, IL3, IL9, IL10, IL13, IL17B, IL19, IL22, IL23A, IL24, IL25, IL28A, and IL31 represent the gene symbols of IL1 $\alpha$, IL2, IL3, IL9, IL10, IL13, IL17 $\beta$, IL19, IL22, IL23 $\alpha$, IL24, IL25, IL28 and IL31, respectively. " $\mathrm{P}<0.05$ and ${ }^{* * *} \mathrm{P}<0.01$. PBMCs, peripheral blood mononuclear cells; PE, pulmonary embolism.

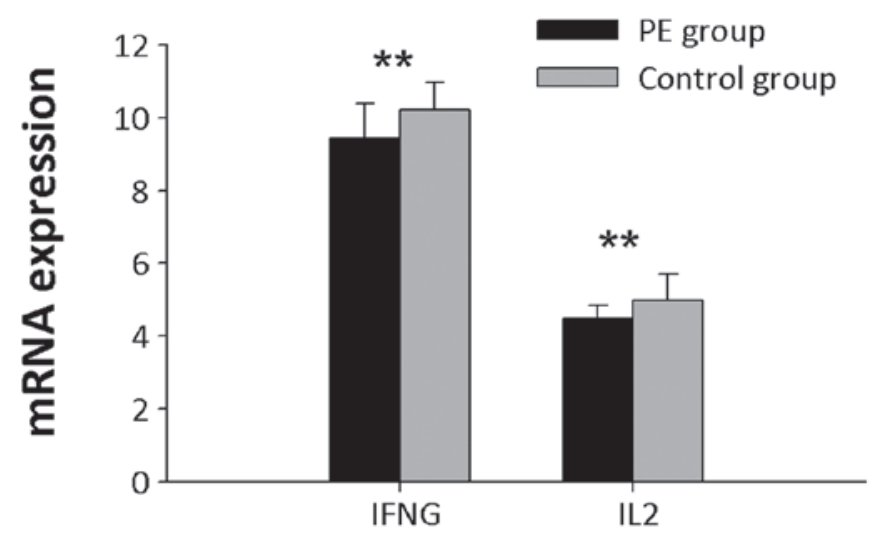

Figure 3. Th1 cytokine mRNA expression levels in PBMCs from PE patients and controls. IFNG and IL2 represent the gene symbols of IFN $\gamma$ and IL2, respectively. ${ }^{*} \mathrm{P}<0.05$ and ${ }^{* *} \mathrm{P}<0.01$. PBMCs, peripheral blood mononuclear cells; PE, pulmonary embolism.

receptor superfamily members $11 \mathrm{~B}, 19$ and 25 , were significantly downregulated compared with controls $(\mathrm{P}<0.05)$.

Colony stimulating factor mRNA expression levels. Six genes encoding colony stimulating factors were detected

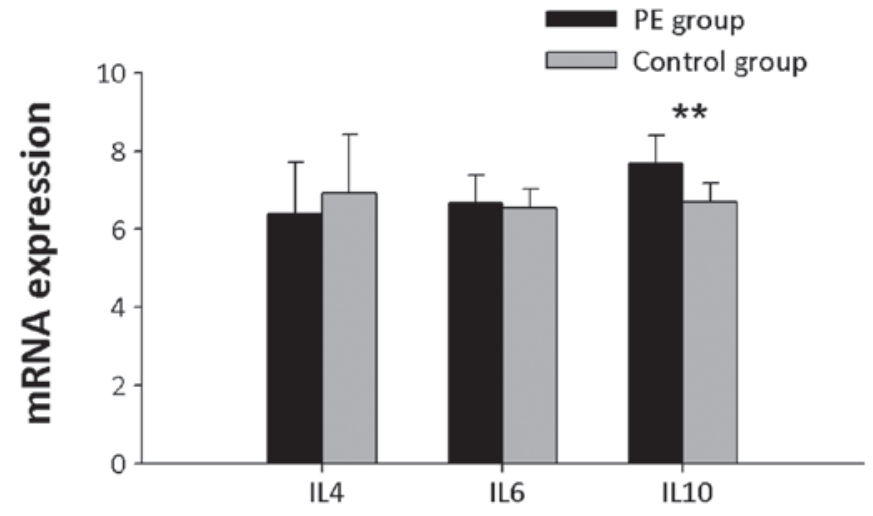

Figure 4. Th2 cytokine mRNA expression levels in PBMCs from PE patients and controls. IL4, IL6 and IL10 represent the gene symbols of IL4, IL6 and IL10, respectively. ${ }^{*} \mathrm{P}<0.05$ and ${ }^{* *} \mathrm{P}<0.01$. PBMCs, peripheral blood mononuclear cells; PE, pulmonary embolism.

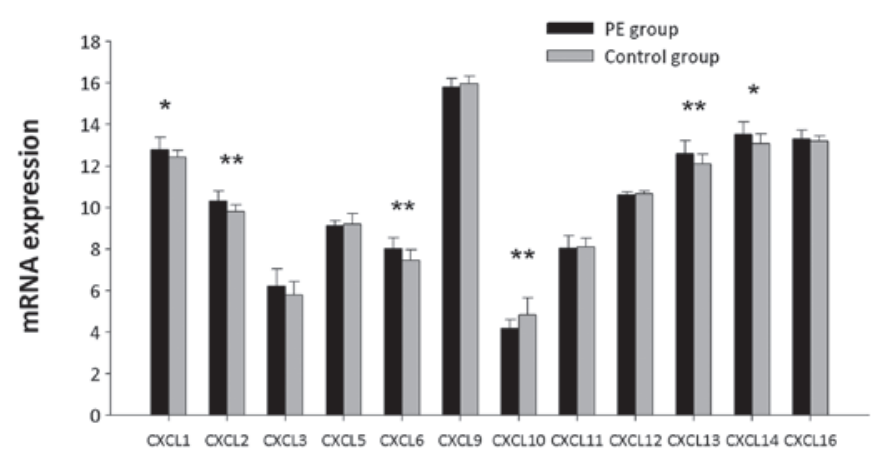

Figure 5. CXC chemokine mRNA expression levels in PBMCs from PE patients and controls. CXCL1, CXCL2, CXCL3, CXCL5, CXCL6, CXCL9, CXCL10, CXCL11, CXCL12, CXCL13, CXCL14 and CXCL16 represent the gene symbols of Cxcl1, Cxcl2, Cxcl3, Cxc15, Cxc16, Cxc19, Cxcl10, Cxcl11, Cxcl12, Cxcl13, Cxcl14 and Cxc116, respectively. ${ }^{*} \mathrm{P}<0.05$ and ${ }^{* * *} \mathrm{P}<0.01$. PBMCs, peripheral blood mononuclear cells; $\mathrm{PE}$, pulmonary embolism.

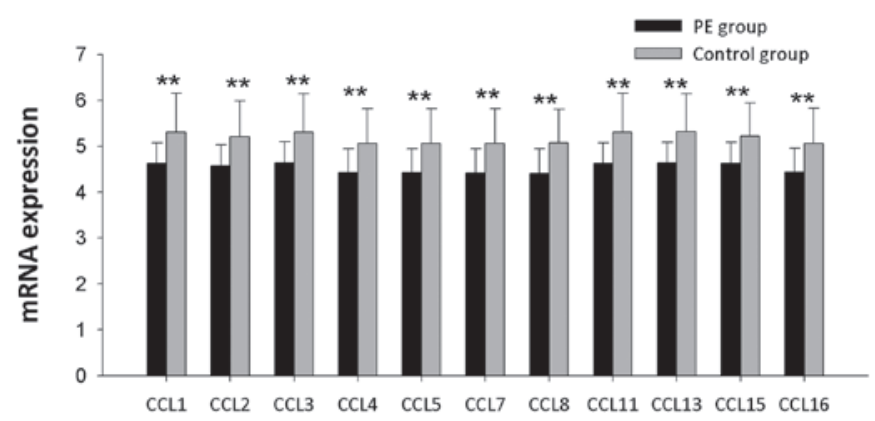

Figure 6. CC chemokine mRNA expression levels in PBMCs from PE patients and controls. CCL1, CCL2, CCL3, CCL4, CCL5, CCL7, CCL8, CCL11, CCL13, CCL15 and CCL16 represent the gene symbols of Ccl1, Ccl2, Ccl3, Cc14, Cc15, Ccl7, Cc18, Ccl11, Cc113, Ccl15 and Ccl16, respectively. " $\mathrm{P}<0.05$ and ${ }^{* *} \mathrm{P}<0.01$. PBMCs, peripheral blood mononuclear cells; PE, pulmonary embolism.

(Fig. 10) and the mRNA expression levels of granulocytemacrophage colony stimulating factor (GM-CSF), granulocyte colony-stimulating factor (G-CSF), erythropoietin (EPO), thrombopoietin (THPO) and mast cell growth factor (KITLG) were significantly lower in PBMCs from PE patients than controls $(\mathrm{P}<0.05)$. 


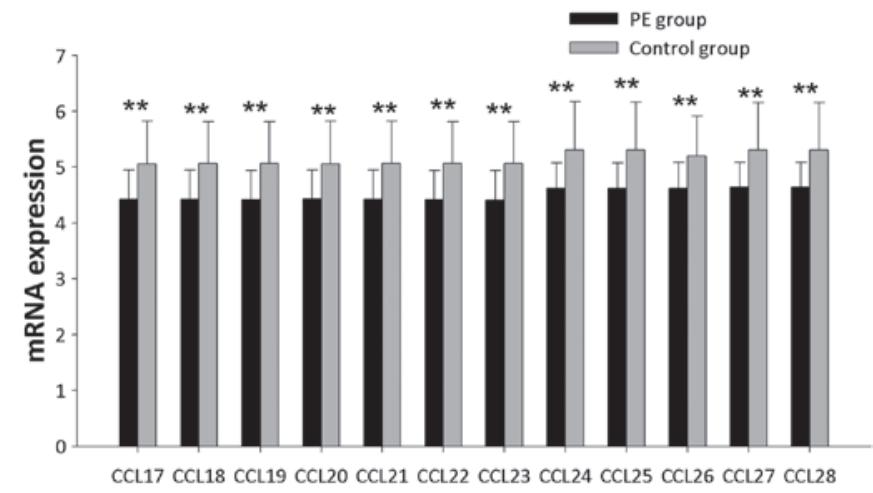

Figure 7. CC chemokine mRNA expression levels in PBMCs from PE patients and controls. CCL17, CCL18, CCL19, CCL20, CCL21, CCL22, CCL23, CCL24, CCL25, CCL26, CCL27 and CCL28 represent the gene symbols of Ccl17, Ccl18, Ccl19, Ccl20, Ccl21, Ccl22, Ccl23, Ccl24, Ccl25, Ccl26, Ccl27 and $\mathrm{Ccl} 28$, respectively. ${ }^{*} \mathrm{P}<0.05$ and ${ }^{* *} \mathrm{P}<0.01$. PBMCs, peripheral blood mononuclear cells; $\mathrm{PE}$, pulmonary embolism.

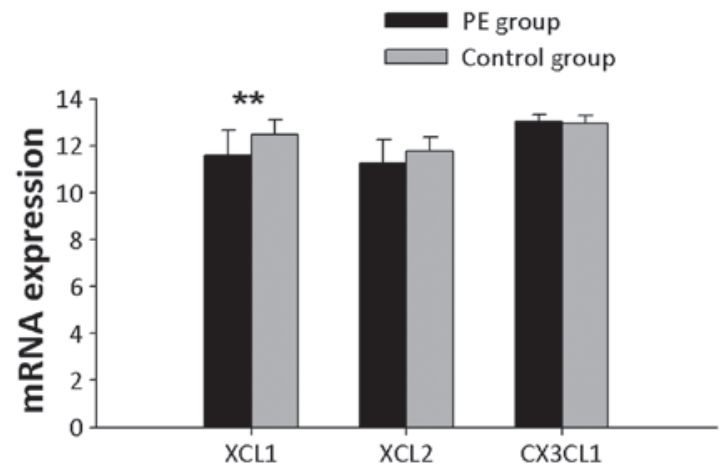

Figure 8. C and CX3C chemokine mRNA expression levels in PBMCs from PE patients and controls. XCL1, XCL2 and CX3CL1 represent the gene symbols of $\mathrm{Xcl1}, \mathrm{Xcl} 2$ and $\mathrm{Cx} 3 \mathrm{cll}$, respectively. $\mathrm{P}<0.05$ and ${ }^{* *} \mathrm{P}<0.01$. PBMCs, peripheral blood mononuclear cells; PE, pulmonary embolism.

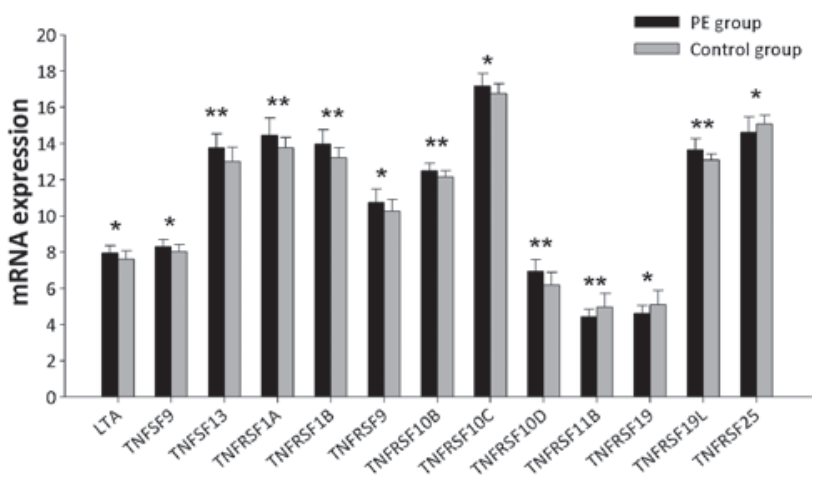

Figure 9. TNF superfamily and TNF receptor superfamily mRNA expression levels which were significantly different in PBMCs from PE patients and controls. LTA, TNFSF9, TNFSF13, TNFRSF1A, TNFRSF1B, TNFRSF9, TNFRSF10B, TNFRSF10C, TNFRSF10D, TNFRSF11B, TNFRSF19, TNFRSF19L, and TNFRSF25 represent the gene symbols of TNF superfamily members 1,9 and 13 , and TNF receptor superfamily members $1 \mathrm{~A}$, 1B, 9, 10B, 10C, 10D, 11B, 19, 19L and 25, respectively. ${ }^{*} \mathrm{P}<0.05$ and ${ }^{* * *} \mathrm{P}<0.01$. PBMCs, peripheral blood mononuclear cells; PE, pulmonary embolism.

Other cytokine mRNA expression levels. We examined eight genes associated with transforming growth factor (TGF), epidermal growth factor (EGF) and vascular endothelial

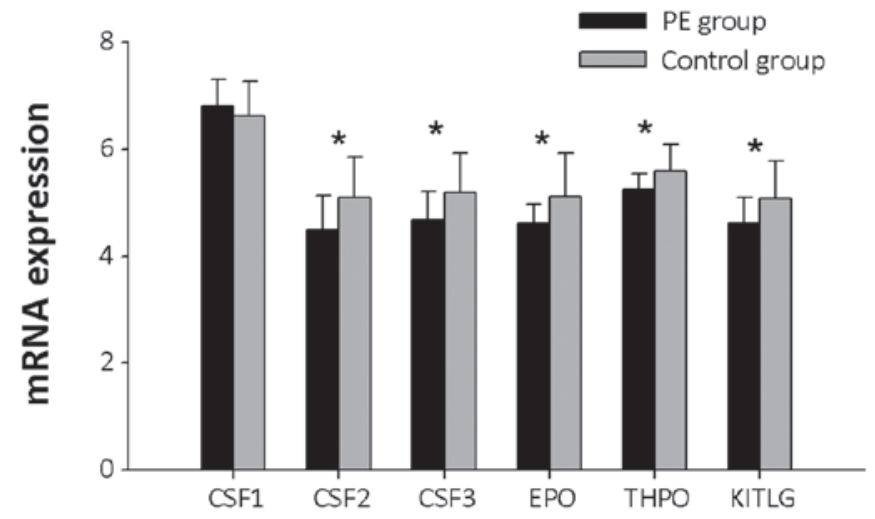

Figure 10. CSF mRNA expression levels in PBMCs from PE patients and controls. CSF1, CSF2, CSF3, EPO, THPO and KITLG represent the gene symbols of macrophage colony stimulating factor, granulocyte-macrophage colony stimulating factor, granulocyte colony stimulating factor, erythropoietin, thrombopoietin and mast cell growth factor, respectively. " $\mathrm{P}<0.05$ and ${ }^{* *} \mathrm{P}<0.01$. PBMCs, peripheral blood mononuclear cells; PE, pulmonary embolism; CSF, colony stimulating factor; EPO, erythropoietin; THPO, thrombopoietin; KITLG, mast cell growth factor.

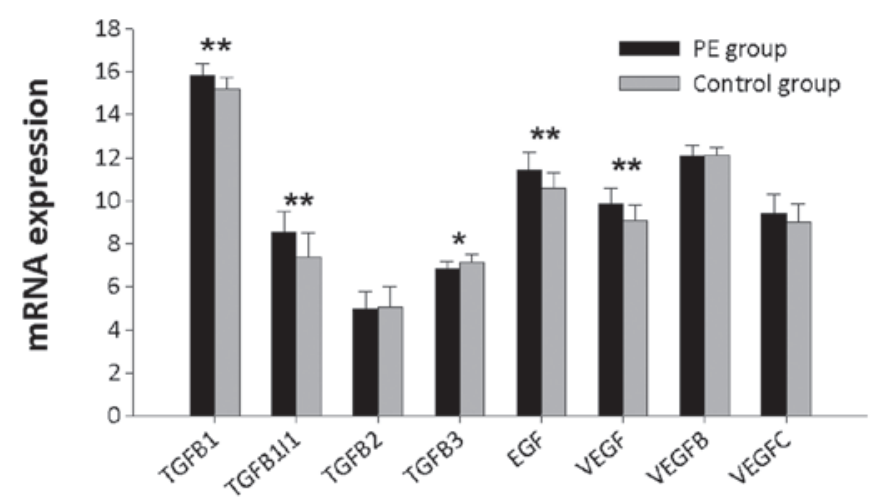

Figure 11. Other cytokine mRNA expression levels in PBMCs from PE patients and controls. TGFB1, TGFB1I1, TGFB2, TGFB3, EGF, VEGF, VEGFB and VEGFC represent the gene symbols of TGF $\beta 1$, TGF $\beta 1$-induced transcript 1, TGF $\beta 2$, TGF $\beta 3$, epidermal growth factor, VEGF, VEGFB and VEGFC, respectively. ${ }^{*} \mathrm{P}<0.05$ and ${ }^{* *} \mathrm{P}<0.01$. PBMCs, peripheral blood mononuclear cells; PE, pulmonary embolism; VEGF, vascular endothelial growth factor; TGF, transforming growth factor; EGF, epidermal growth factor.

growth factor (VEGF) (Fig. 11). The mRNA expression levels of TGF $\beta 1$, TGF $\beta 1$-induced transcript 1, EGF and VEGF were significantly upregulated $(\mathrm{P}<0.01)$, whereas TGF $\beta 3 \mathrm{mRNA}$ was significantly downregulated $(\mathrm{P}<0.05)$ in $\mathrm{PBMCs}$ from PE patients compared with controls.

\section{Discussion}

IFNs are classified into two major groups: type I and type II. Type I IFNs include IFN $\alpha$, IFN $\beta$, IFN $\varepsilon$, IFN $\omega$ and IFNא (4). We detected type I IFN mRNAs in the PE patients compared with controls. We demonstrated that IFN $\alpha 5$, IFNa6, IFN $\alpha 8$, IFN $\alpha 14$, IFN $\kappa$, IFN $\omega 1$ and IFNe1 mRNA expression levels were significantly lower in PE patients compared with controls. The type I IFNs possess antiviral, antiproliferative and immunomodulatory effects (5). It has been reported that IFN $\alpha / \beta$ receptor knockout mice are susceptible to viral infection (6). 
Mice treated with antibodies against IFN $\alpha / \beta$ may be lethally infected with filoviruses (7). Thus, our results suggest that PE patients are susceptible to viral infection. In this study, we also examined IFN $\gamma$ mRNA expression levels which were observed to be significantly downregulated in PE patients compared with controls. IFN $\gamma$ is important in the defence against intracellular bacteria (Mycobacterium, Listeria) and parasites (Leishmania) (8). A caudal vena cava thrombus in a dog with leishmaniasis has been reported (9). It has also been demonstrated that three patients with pulmonary tuberculosis suffered from PTE or DVT (10). Therefore, our results show that PE patients are susceptible to intracellular bacteria and parasites.

T-helper lymphocytes can be divided into Th1 and Th2 subsets based on their functional abilities and the profile of the cytokines they produce (11). Th1 and Th2 cells express different cytokines: Th1 cells produce IL2, IL12 and IFN $\gamma$, whereas Th2 cells produce IL4, IL5, IL6, IL10 and IL13 (12). Th1 cells promote cellular immunity, while Th2 cells induce humoral immunity and upregulate antibody production (13). The shift in Th1/Th2 balance leads to immune dysfunction. Our results demonstrated the presence of lower mRNA expression levels of Th1 cytokines (IFN $\gamma$ and IL2) and higher mRNA expression levels of Th2 cytokine (IL10) in PE patients, suggesting a shift towards Th2 dominance in PE patients. Thus, our results suggest that an immune dysfunction in PE patients may exist.

Chemokines can be classified into four classes; CXC, $\mathrm{CC}, \mathrm{C}$ and $\mathrm{CX} 3 \mathrm{C}$ according to their structure. Previous studies have shown that a polymorphonuclear (PMN) activation in thrombosis is present, and that PMN activation may be important in the etiologies of thrombosis $(14,15)$. CXC chemokines possess a potent chemotactic activity on neutrophils. Twelve genes encoding $\mathrm{CXC}$ chemokines and mRNA expression levels of $\mathrm{Cxcl1}, \mathrm{Cxcl} 2, \mathrm{Cxcl6}, \mathrm{Cxcl13}$ and $\mathrm{Cxcl14}$ were significantly upregulated in $\mathrm{PE}$ patients compared with controls, suggesting that a high expression of chemokines may induce neutrophil aggregation. The CC chemokines RANTES/CCL5, MIP-1 $\alpha /$ CCL3 and MIP-1 $\beta /$ CCL4 produced by CD8+ T cells induce the inhibition of human immunodeficiency virus (HIV) (16). During chronic viral infection, virus-specific CD8 T cells generated low levels of cytokines and the cytotoxic ability of CD8 T cells was decreased in RANTES knockout mice (17). Deficiency of MIP-1 $\alpha$ led to the recovery of higher levels of infectious viruses in comparison with fully competent mice in paramyxovirus infection (18). During mouse hepatitis virus infection, the cytokine production and cytolytic activity of CD8+ T cells were reduced in CCL3 knockout mice (19). Our results demonstrate the presence of lower mRNA expression levels of $\mathrm{Ccl} 3, \mathrm{Ccl} 4$ and $\mathrm{Ccl} 5$ in PE patients, which suggests that a reduced antiviral ability in PE patients may exist.

The interaction of costimulatory receptor CD137 (TNF receptor superfamily, member 9) and its ligand CD137L (TNF superfamily, member 9), which is involved in the TNF superfamily, is important in immune regulation (20). Blockade of the CD137-CD137L pathway significantly reduced the percentage of CD3+ CD8+ $\mathrm{T}$ cells in mice transplanted with donor bone marrow cells plus primary MLC spleen T cells (21). It has been reported that 4-1BB (also known as CD137)-deficient mice have enhanced susceptibility to Listeria monocytogenes (intracellular bacteria) infections (22). It has also been shown that the cytotoxic lymphocyte (CTL) response to the influenza virus was reduced in mice lacking 4-1BBL (23). In this study, mRNA expression levels of CD137 and CD137L were upregulated in PE patients, suggesting that PE patients may be more susceptible to infection. TNF may interfere with viral replication in several ways (24). TNF has two receptors, TNF receptor 1 (TNFR1) and 2 (TNFR2) (24). The TNF/TNFR pathway is important in the induction of apoptosis (25). It has been demonstrated that TNFR1 and TNFR2 induced evident apoptosis, including CD4 and CD8 T cells during HIV infection (26). In our study, mRNA expression levels of TNFR1 and TNFR2 were significantly greater in PE patients than controls. Thus, we hypothesize that peripheral T-cell apoptosis induced by TNFR1 and TNFR2 may exist, leading to a decreased immune function and enhanced susceptibility to virus expression in PE patients.

GM-CSF is important to the immune system. GM-CSF is capable of affecting the balance of Th1/Th2. It has been reported that mice lacking GM-CSF were not able to control an aerosol-delivered infection with Mycobacterium tuberculosis and died from severe necrosis in the lungs as it was not able to express a Th1 response (27). In this study, mRNA expression levels of GM-CSF were significantly downregulated in PE patients, suggesting that there may be decreased immune function and a reduced antipathogenic micro-organism ability in PE patients.

VEGF-A (also known as VEGF) is an important member of the VEGF family. PE-induced endothelium injury is important in the sequelae following PE (28). In this study, mRNA expression levels of VEGF in PE patients were significantly higher than controls, suggesting that the level of VEGF mRNA may increase compensatively to promote endothelial cell repair and vascular remodeling following PE-induced endothelium injury. Three types of isoform have been identified in mammals; TGF $\beta 1$, TGF $\beta 2$ and TGF $\beta 3$ (29). All three isoforms have similar properties in vitro, however, TGF $\beta 1$ is dominantly expressed in the immune system (30). It has been reported that the level of TGF $\beta 1$ increases during cytomegalovirus and hepatitis B virus infection $(31,32)$. It has been shown that in HIV infection, TGF $\beta$ is capable of suppressing the cellular immune response and the humoral immune response (33). In this study, mRNA expression levels of TGF were upregulated significantly in PE patients, suggesting that a decreased immune function in PE patients may exist and this disease may be associated with viral infections.

From the characteristics of a variety of cytokine mRNA expression levels in PE patients, we conclude that the immune function and the ability of clearing viruses, intracellular bacteria and parasites are reduced in PE patients. Previously, we reported downregulated gene expression associated with NK and T cells in patients with PE (34), and the number of CD3+ and CD8+ T cells were significantly downregulated in patients with APE and CTEPH (2,3). In this study, we detected and analyzed the mRNA expression levels of cytokines in patients with $\mathrm{PE}$ and conclude that an immune dysfunction in PE patients exists, which is similar with the results of previously performed cytology tests. Our results may provide evidence for the etiology and pathogenesis of VTE. 


\section{Acknowledgements}

This study was supported by the '12th Five Year' National Science and Technology Supporting Program (2011BAI11B16).

\section{References}

1. Heit JA: The epidemiology of venous thromboembolism in the community. Arterioscler Thromb Vasc Biol 28: 370-372, 2008

2. Wang L, Song H, Gong Z, Duan Q and Liang A: Acute pulmonary embolism and dysfunction of CD3+ CD8+ T cell immunity. Am J Respir Crit Care Med 184: 1315, 2011.

3. Haoming S, Lemin $\mathrm{W}$, Zhu G, et al: T cell-mediated immune deficiency or compromise in patients with CTEPH. Am J Respir Crit Care Med 183: 417-418, 2011.

4. Sang Y, Rowland RR, Hesse RA and Blecha F: Differential expression and activity of the porcine type I interferon family. Physiol Genomics 42: 248-258, 2010.

5. Day SL, Ramshaw IA, Ramsay AJ and Ranasinghe C: Differential effects of the type I interferons alpha4, beta, and epsilon on antiviral activity and vaccine efficacy. J Immunol 180 7158-7166, 2008.

6. Abbas AK and Lichtman AH (eds): Cellular and Molecular Immunology. 5th edition. Saunders, Philadelphia, PA, pp562, 2005

7. Bray M: The role of the Type I interferon response in the resistance of mice to filovirus infection. J Gen Virol 82: 1365-1373, 2001.

8. van den Broek MF, Müller U, Huang S, Zinkernagel RM and Aguet M: Immune defence in mice lacking type I and/or type II interferon receptors. Immunol Rev 148: 5-18, 1995.

9. Font A and Closa JM: Ultrasonographic localization of a caudal vena cava thrombus in a dog with leishmaniasis. Vet Radiol Ultrasound 38: 394-396, 1997.

10. Komazaki Y, Sakakibara Y, Sakashita H, Miyazaki Y and Inase N: Pulmonary thromboembolism with pulmonary tuberculosis. Kekkaku 86: 717-722, 2011 (In Japanese).

11. Constant SL and Bottomly K: Induction of Th1 and Th2 CD4+ T cell responses: the alternative approaches. Annu Rev Immunol 15: 297-322, 1997.

12. Romagnani S: Biology of human TH1 and TH2 cells. J Clin Immunol 15: 121-129, 1995.

13. Kidd P: Th1/Th2 balance: the hypothesis, its limitations, and implications for health and disease. Altern Med Rev 8: 223-246, 2003 .

14. LoPresti R, Ferrara F, Canino B, Montana M and Caimi G: Deep venous thrombosis: leukocyte rheology at baseline and after in vitro activation. Haemostasis 30: 168-173, 2000.

15. Esposito CJ, Popescu WM, Rinder HM, Schwartz JJ, Smith BR and Rinder CS: Increased leukocyte-platelet adhesion in patients with graft occlusion after peripheral vascular surgery. Thromb Haemost 90: 1128-1134, 2003

16. Cocchi F, DeVico AL, Garzino-Demo A, Arya SK, Gallo RC and Lusso P: Identification of RANTES, MIP-1 alpha, and MIP-1 beta as the major HIV-suppressive factors produced by CD8+ T cells. Science 270: 1811-1815, 1995.

17. Crawford A, Angelosanto JM, Nadwodny KL, Blackburn SD and Wherry EJ: A role for the chemokine RANTES in regulating CD8 T cell responses during chronic viral infection. PLoS Pathog 7: e1002098, 2011.

18. Domachowske JB, Bonville CA, Gao JL, Murphy PM, Easton AJ and Rosenberg HF: The chemokine macrophage-inflammatory protein-1 alpha and its receptor CCR1 control pulmonary inflammation and antiviral host defense in paramyxovirus infection. J Immunol 165: 2677-2682, 2000.
19. Trifilo MJ, Bergmann CC, Kuziel WA and Lane TE: CC chemokine ligand 3 (CCL3) regulates CD8(+)-T-cell effector function and migration following viral infection. J Virol 77: 4004-4014, 2003.

20. Vinay DS and Kwon BS: Immunotherapy targeting 4-1BB and its ligand. Int J Hematol 83: 23-28, 2006.

21. Li CH, Xu KL, Pan XY and Du B: Study on control of graft-versus-host disease by blocking CD137-CD137L ligand costimulatory pathway in mice. Zhonghua Xue Ye Xue Za Zhi 28: 93-97, 2007 (In Chinese).

22. Lee SC, Ju SA, Pack HN, et al: 4-1BB (CD137) is required for rapid clearance of Listeria monocytogenes infection. Infect Immun 73: 5144-5151, 2005.

23. DeBenedette MA, Wen T, Bachmann MF, et al: Analysis of 4-1BB ligand (4-1BBL)-deficient mice and of mice lacking both 4-1BBL and CD28 reveals a role for 4-1BBL in skin allograft rejection and in the cytotoxic $\mathrm{T}$ cell response to influenza virus. J Immunol 163: 4833-4841, 1999.

24. Herbein G and O'Brien WA: Tumor necrosis factor (TNF)-alpha and TNF receptors in viral pathogenesis. Proc Soc Exp Biol Med 223: 241-257, 2000.

25. Micheau O and Tschopp J: Induction of TNF receptor I-mediated apoptosis via two sequential signaling complexes. Cell 114: 181-190, 2003.

26. de Oliveira Pinto LM, Garcia S, Lecoeur H, Rapp C and Gougeon ML: Increased sensitivity of T lymphocytes to tumor necrosis factor receptor 1 (TNFR1)- and TNFR2-mediated apoptosis in HIV infection: relation to expression of $\mathrm{Bcl}-2$ and active caspase-8 and caspase-3. Blood 99: 1666-1675, 2002.

27. Gonzalez-Juarrero M, Hattle JM, Izzo A, et al: Disruption of granulocyte macrophage-colony stimulating factor production in the lungs severely affects the ability of mice to control Mycobacterium tuberculosis infection. J Leukoc Biol 77: 914-922, 2005

28. Wan J, Lu LJ, Miao R, et al: Alterations of bone marrow-derived endothelial progenitor cells following acute pulmonary embolism in mice. Exp Biol Med (Maywood) 235: 989-998, 2010.

29. Govinden R and Bhoola KD: Genealogy, expression, and cellular function of transforming growth factor-beta. Pharmacol Ther 98: 257-265, 2003

30. Li MO, Wan YY, Sanjabi S, Robertson AK and Flavell RA: Transforming growth factor-beta regulation of immune responses. Annu Rev Immunol 24: 99-146, 2006.

31. Haagmans BL, Teerds KJ, van den Eijnden-van Raaij AJ, Horzinek MC and Schijns VE: Transforming growth factor beta production during rat cytomegalovirus infection. J Gen Virol 78 (Pt 1): 205-213, 1997.

32. Yoo YD, Ueda H, Park K, et al: Regulation of transforming growth factor-beta 1 expression by the hepatitis B virus (HBV) $\mathrm{X}$ transactivator. Role in HBV pathogenesis. J Clin Invest 97: 388-395, 1996

33. Kekow J, Wachsman W, McCutchan JA, et al: Transforming grow th factor-beta and suppression of humoral immune responses in HIV infection. J Clin Invest 87: 1010-1016, 1991.

34. Gong Z, Liang AB, Wang LM, et al: The expression and significance of immunity associated genes mRNA in patients with pulmonary embolism. Zhonghua Nei Ke Za Zhi 48: 666-669, 2009 (In Chinese) 